\title{
Aorta Fluorescence Imaging by Using Confocal Microscopy
}

\author{
Chun-Yang Wang, ${ }^{1,2}$ Jui-che Tsai, ${ }^{3}$ Ching-Cheng Chuang, ${ }^{4}$ \\ Yao-Sheng Hsieh, ${ }^{2}$ and Chia-Wei Sun ${ }^{1,2}$ \\ ${ }^{1}$ Biophotonics Interdisciplinary Research Center and Institute of Biophotonics, National Yang-Ming University, \\ Taipei 11221, Taiwan \\ ${ }^{2}$ Department of Photonics, National Chiao Tung University, Hsinchu 30010, Taiwan \\ ${ }^{3}$ Graduate Institute of Photonics and Optoelectronics and Department of Electrical Engineering, National Taiwan University, \\ Taipei 10617, Taiwan \\ ${ }^{4}$ Institute of Biomedical Engineering, National Taiwan University, Taipei 10051, Taiwan
}

Correspondence should be addressed to Chia-Wei Sun, chiaweisun@ym.edu.tw

Received 17 March 2011; Accepted 3 May 2011

Academic Editors: E. A. Bocchi, M. Saeed, and C. Vassalle

Copyright (C) 2011 Chun-Yang Wang et al. This is an open access article distributed under the Creative Commons Attribution License, which permits unrestricted use, distribution, and reproduction in any medium, provided the original work is properly cited.

The activated leukocyte attacked the vascular endothelium and the associated increase in VEcadherin number was observed in experiments. The confocal microscopic system with a prism-based wavelength filter was used for multiwavelength fluorescence measurement. Multiwavelength fluorescence imaging based on the VEcadherin within the aorta segment of a rat was achieved. The confocal microscopic system capable of fluorescence detection of cardiovascular tissue is a useful tool for measuring the biological properties in clinical applications.

\section{Introduction}

In recent years, the confocal microscopic technique has had an important role in biology and medicine, especially for fluorescence imaging of tissues [1-6]. Compared with the conventional optical microscopy, the confocal microscopy provides more advantages, including controllable depth of field, elimination of out-of-focus information, and the capability to collect serial optical sections from thick specimens. Recently, fibered confocal fluorescence microscopy was combined in endoscopic imaging system [7-9]. Confocal endomicroscope aims at providing to the clinician optical biopsies, that is, in vivo microscopic imaging of a living tissue. Such systems have been successfully applied to the in vivo explorations of the human skin, cervix, and oral cavity, as well as to the endomicroscopic imaging of the gastric and colonic mucosa and biliary tract. Lately, the microscopic imaging was also achieved in the proximal and distal respiratory systems [10].

Atherosclerosis may occur in the arteries of the brain, heart, kidneys, vital organs, arms, and legs. It is the major cause for myocardial infarction, stroke, and peripheral vascular diseases. It is also the leading cause for illness and deaths in the United States and other developed countries [11]. Atherosclerosis has been studied extensively with various animal models [12]. The degree of lowdensity lipoprotein- (LDL-) induced leukocyte adhesion to endothelium is considered to be an important factor of developing atherosclerosis, and one of the earliest stages of atherogenesis is endothelial dysfunction [13]. In animal study using rats, the aorta endothelium has a relatively small thickness of $\sim 100 \mu \mathrm{m}$, which makes it difficult to obtain images under a conventional microscope. The confocal fluorescence microscope is a suitable alternative for acquiring sectioned images. It has observed LDL-induced leukocyte adhesion to endothelium.

Simultaneous multiwavelength fluorescence screening, for example, in observing certain proteins labeled with different fluorescent dyes [14-16], has attracted increasing attention [17]. Multiple excitation wavelengths are emitted from various lasers. The multiwavelength fluorescence signals can be measured by detectors with optical filters. In a conventional confocal fluorescence microscope, normally a filter wheel with chosen wavelength ranges was used $[18,19]$. 


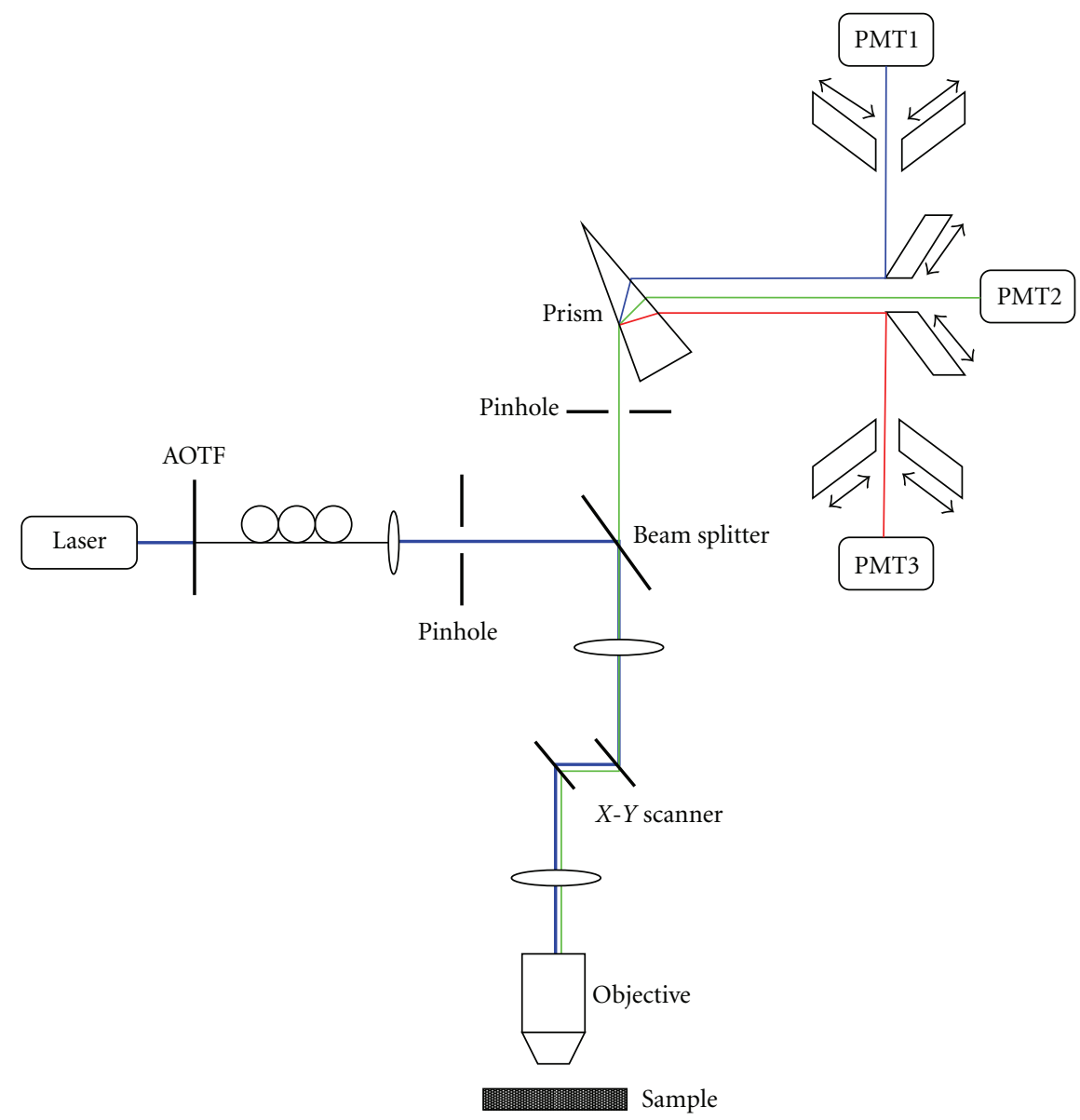

Figure 1: The schematic setup of our confocal microscope. The laser beam for excitation is incident upon the sample. With the prism, the signal splits into three wavelength ranges, each of which is detected by one PMT.

However, with the choice of dyes widening, the design needs be modified for multiwavelength measurement as detection based on rotating the filter wheel for different wavelengths with longer time-consuming and higher cost. Therefore, an adjustable design for optical filters is needed to involve the wavelength range of fluorescence [20-22].

In this paper, a confocal spectral microscope was used to measure the multiwavelength fluorescence images from the cut-open rat aorta. Both auto- and extra-fluorescence detections have been achieved in experiments. Controllable prism-based wavelength filters are incorporated into our microscopic system to obtain the multiwavelength fluorescence images [23]. The activated leukocyte attacks the vascular endothelium and the associated increase in VEcadherin number is observed in the experiments.

\section{Experiments}

In our study, a confocal microscopic system (Leica TCS SP2) was used to obtain the multiwavelength fluorescence images of aorta samples. An Ar-Kr laser of $488 \mathrm{~nm}$ and a He-Ne laser of $543 \mathrm{~nm}$ are the excitation light sources. Figure 1 shows the schematic setup of our confocal microscopic system. The laser beam passes though an acoustic optical tunable filter (AOTF) and is coupled into a fiber bundle. The AOTF is used to control the excitation intensity to avoid the photobleaching of the sample. The laser beam is then reflected to the sample by the dichroic beam splitter. The signal from sample is collected with an objective, passes though the dichroic beam splitter, and is finally incident on a prism. With the prism, the signal splits into three wavelength ranges, each of which is detected by one PMT [22]. The $X-Y$ scanner is used to scan the sample for $2 \mathrm{D}$ imaging. The cut-open aorta tissue of a rat is prepared for in vitro measurement. The Ar-Kr laser excites the green autofluorescence from proteins in tissue, while the He-Ne laser stimulates the red extrafluorescence from the cells of rat's endothelium labeled with dye alexa568 conjugated lectin GS-IB4. The scanning line rate is $400 \mathrm{~Hz}$ in the experiment.

The fluorescence images of rat's aorta segments were detected by a confocal microscope with the prism-based filter for the wavelength selection. In human bodies, the cholesterol cannot dissolve in blood. Lipoproteins play an important role in the cholesterol transport between cells. There are two kinds of lipoproteins: high-density lipoprotein (HDL) and low-density lipoprotein (LDL). HDL carries cholesterol away from the blood to prevent the heart disease. LDL, so-called "bad cholesterol", builds up in the inner walls of the arteries. LDL causes the atherosclerosis when it combines with other substance and then induces the plaque 


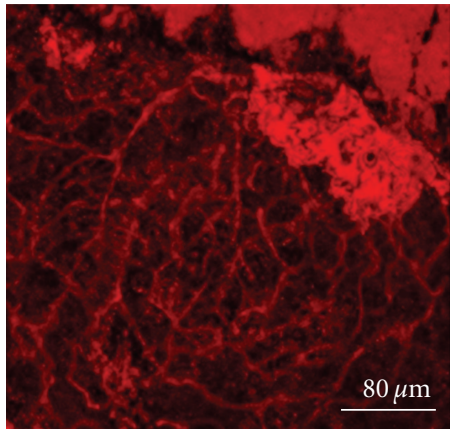

(a)

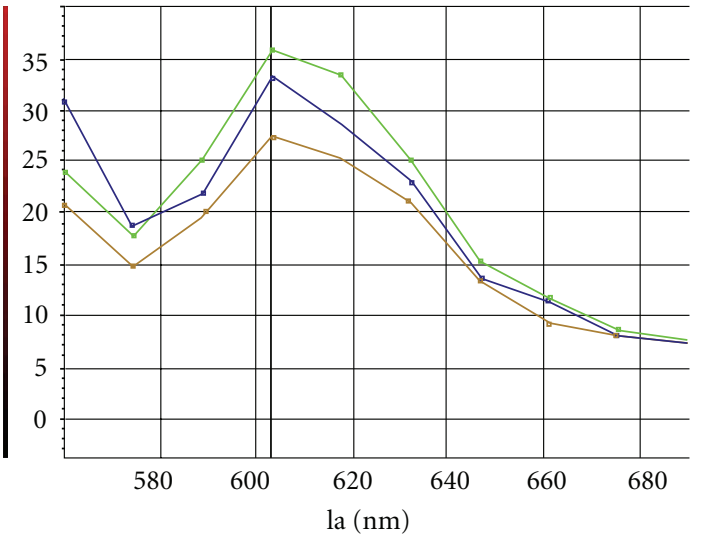

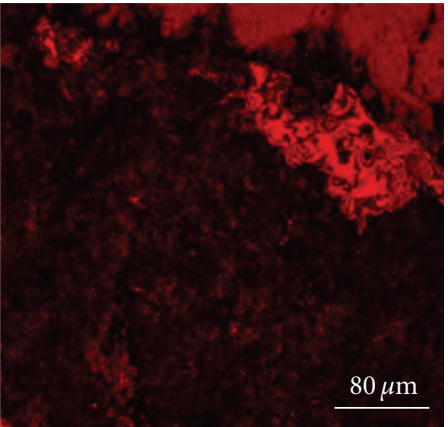

(b)

(d)

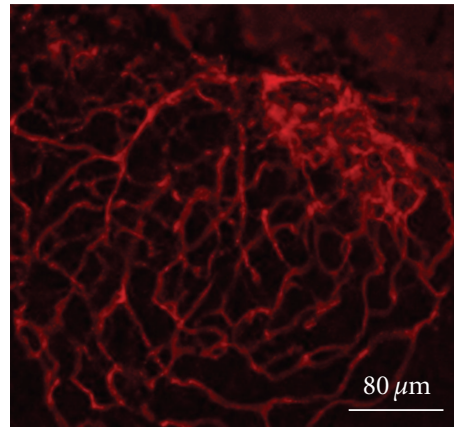

(c)

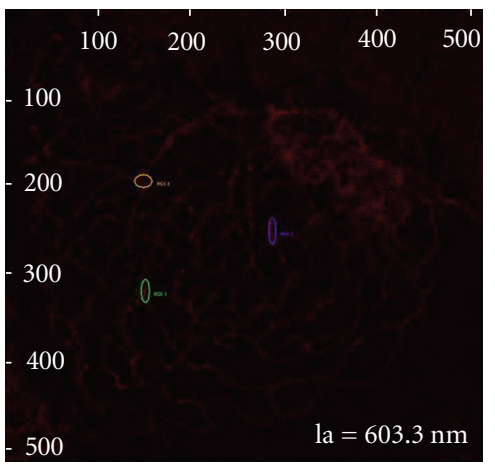

FIgURE 2: Red fluorescence images of the rat endothelial cells excited by He-Ne laser. The wavelength ranges are (a) 555-700 nm, (b) 555$575 \mathrm{~nm}$, and (c) 600-630 nm, respectively. (d) The fluorescence spectra: each colored curve represents the data taken at the spot marked with the circle of that color.

generation in arteries $[24,25]$. Thus, LDL indicates either leukocyte-endothelium interaction or platelet aggregation in the blood stream.

In this experiment, we observed the in vitro fluorescence images of a cut-open aorta of the rat $[26,27]$. The relation between the VEcadherin and the activated leukocyte is verified experimentally. The samples are prepared under three distinct conditions: (1) the leukocyte is treated with $10 \mathrm{mg} / \mathrm{mL}$ native LDL solution and then mixed with the buffer-treated endothelial cells; (2) the leukocyte is treated with buffer and mixed with the endothelial cells, which are treated with $10 \mathrm{mg} / \mathrm{mL}$ native LDL solution; (3) the leukocyte and the endothelial cells are both treated with $10 \mathrm{mg} / \mathrm{mL}$ native LDL solution.

\section{Results and Discussion}

The red fluorescence images of endothelial cells excited by He-Ne laser are shown in Figure 2. Figure 2(a) is the image with the measured fluorescence range spanning from $555 \mathrm{~nm}$ to $700 \mathrm{~nm}$, the TRITC fluorochrome range. In Figures 2(b) and $2(\mathrm{c})$, the measured wavelength ranges of fluorescence are $555-575 \mathrm{~nm}$ and $600-630 \mathrm{~nm}$, respectively. Figure 2(d) shows the fluorescence spectra of 3 selected spots, each marked with a distinct color. The spectral range is 560$700 \mathrm{~nm}$ at $20-\mathrm{nm}$ wavelength intervals. The peak value of each fluorescence spectrum is about $605 \mathrm{~nm}$. Obviously,
Figure 2(d) shows the power of spectrum tail form incident $\mathrm{He}-\mathrm{Ne}$ laser is larger than the fluorescence signal, thus it blurred the red fluorescence images. As a result, Figures 2(a) and 2(b) are the background images due to the backscattered photons of He-Ne laser. Therefore, we select optimal wavelength range in Figure 2(c) and it indicates the pure red fluorescence image from endothelial cells.

The vascular endothelial cadherin (VECAD), the labeled protein, increases in number after the samples have been treated with LDL solution. The VECAD is an endothelialspecific cadherin localized at the intercellular junctions [28]. It could be associated with atherosclerotic lesions by endothelial cells and blood vessels forming [29]. The green fluorescence images from the labeled protein are shown in Figures 3(a)-3(c). The measured fluorescence range of Figure $3(\mathrm{a})$ is $500-540 \mathrm{~nm}$, the FITC fluorochrome range. In Figures $3(\mathrm{~b})$ and $3(\mathrm{c})$, the measured wavelength ranges are 500-505 $\mathrm{nm}$ and 515-535 nm, respectively. The fluorescence spectra with a wavelength range of $510-540 \mathrm{~nm}$ at $7.5-\mathrm{nm}$ intervals are shown in Figure $3(\mathrm{~d})$. The peak value of each spectrum is about $520 \mathrm{~nm}$. Again, the spectrum tail from the Ar-Kr laser blurred the excited green fluorescence signal. Therefore, the 515-535 $\mathrm{nm}$ of measured wavelength ranges is selected and the real green fluorescence signal appears in Figure 3(c). The background signals due to the Ar-Kr laser fringes are shown in Figures 3(a) and 3(b). 


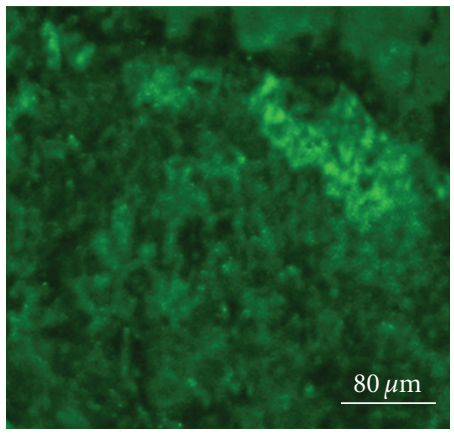

(a)

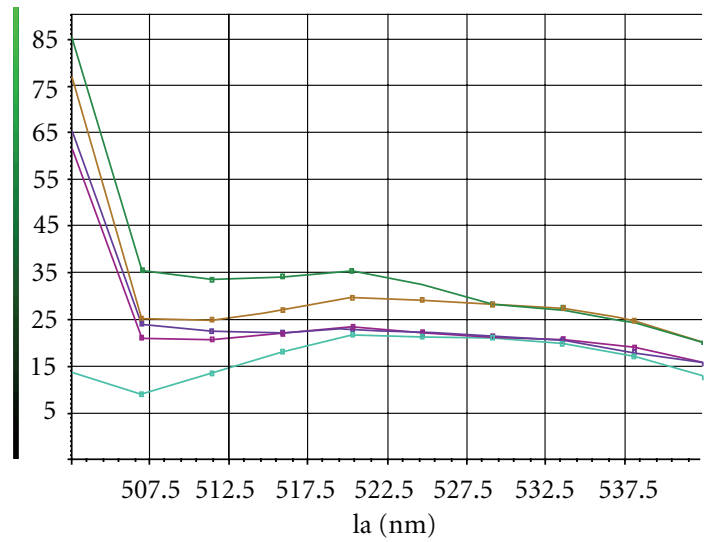

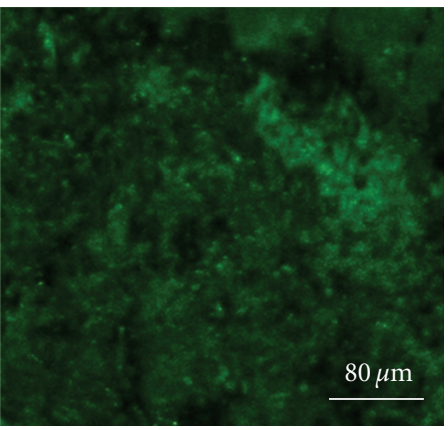

(b)

(d)

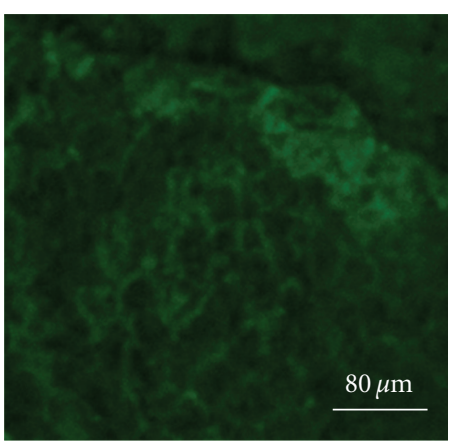

(c)

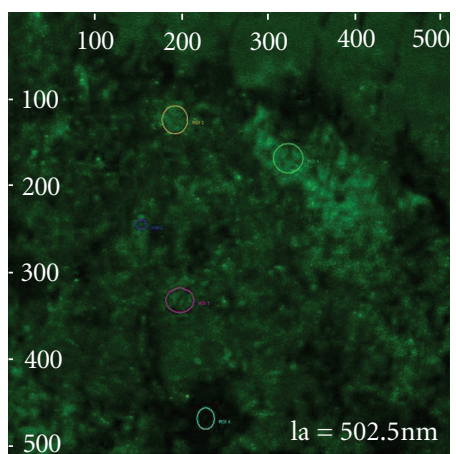

FIgURE 3: Green fluorescence images of the labeled protein excited by the Ar-Kr laser. The wavelength ranges are (a) 500-540 nm, (b) 500$505 \mathrm{~nm}$, and (c) 515-535 nm, respectively. (d) The fluorescence spectra: each colored curve represents the data taken at the spot marked with the circle of that color.

The filter wheel with fixed wavelength range, for example, FITC, TRITC, and so forth, are not good enough for florescence imaging. In order to increase the image contrast as well as the signal-to-noise ratio and to avoid the spectral cross talk, an adjustable wavelength filter is a more appropriate option. Adopting the prism-based filter in the microscopic system could be a helpful tool to analyze fluorescence images immediately without the postprocessing or image reconstruction.

Figure 4 shows the fluorescence images of the cutopen rat aorta treated with $10 \mathrm{mg} / \mathrm{mL}$ LDL solution and/or buffer solution. The wavelength ranges of the measured fluorescence are 600-630 and 515-535 $\mathrm{nm}$ for the red and green images, respectively. The first symbol of each label in Figure 4 stands for the treatment of leukocyte, and the second symbol represents that of the aorta segment. L stands for treatment with $10 \mathrm{mg} / \mathrm{mL}$ native LDL solution, while C means treatment with buffer solution.

In Figure 4(a), the leukocyte and endothelial cells do not treat with any extra solution. There is no green autofluorescence signal, which means no VECAD exists in the sample. In Figures 4(b)-4(d), with the leukocyte and/or rat's aorta segment treated with $10 \mathrm{mg} / \mathrm{mL}$ LDL solution, green autofluorescence is detected, meaning the increase in VECAD number. As mentioned before, the LDL solution activates the leukocyte to attack the rat's aorta endothelial cells and the VECAD number increases. There is then a rise of the possibility of atherosclerosis, which affects the arteries of the brain, heart, kidneys, vital organs, arms, and legs in human bodies

\section{Conclusion}

In this paper, we have demonstrated the measurement of multiwavelength fluorescence of the cut-open rat aorta using a Leica TCS SP2 confocal fluorescence microscope. The experimental results show that an adjustable wavelength filter is suitable for imaging optimization. The spectral range of $350-850 \mathrm{~nm}$ is wide enough for various kinds of fluorescence measurement. Using the $\mathrm{Ar}-\mathrm{Kr}$ laser for green autofluorescence excitation and the $\mathrm{He}-\mathrm{Ne}$ laser for red extra-fluorescence excitation achieves the multicolor images. The prism-based filter provides a flexible selection of wavelength pass range. The optimization of wavelength pass range suppresses noises from the excitation laser fringes, improving the contrast and signal-to-noise ratio.

In this experiment, the results show that the leukocytes attack the aorta endothelial cells after treatment with $10 \mathrm{mg} / \mathrm{mL}$ LDL solution, leading to the increase in number of VECAD. VECAD plays an important role in endothelial cell physiology. The cell contact regulates angiogenesis by controlling endothelial cell adhesion and migration and induces the generation of VECAD under a specific condition [30]. 


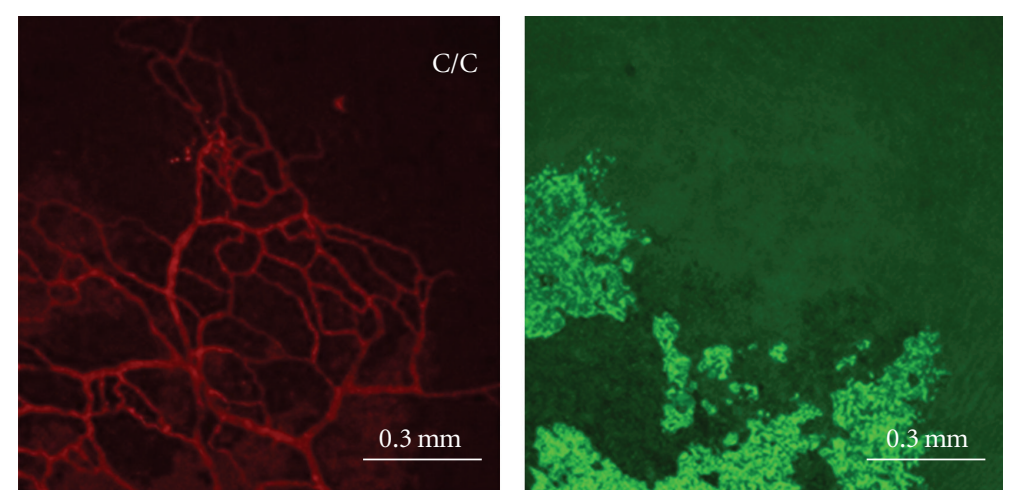

(a)
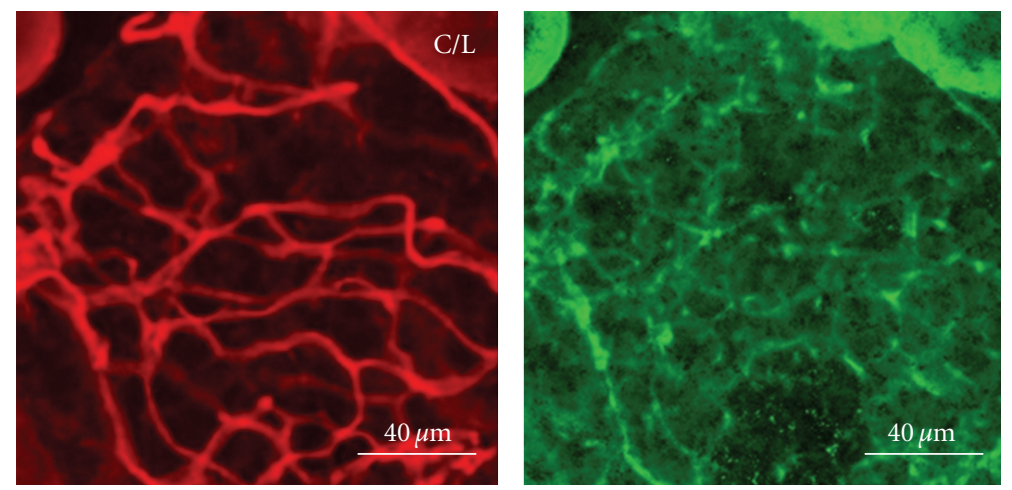

(b)
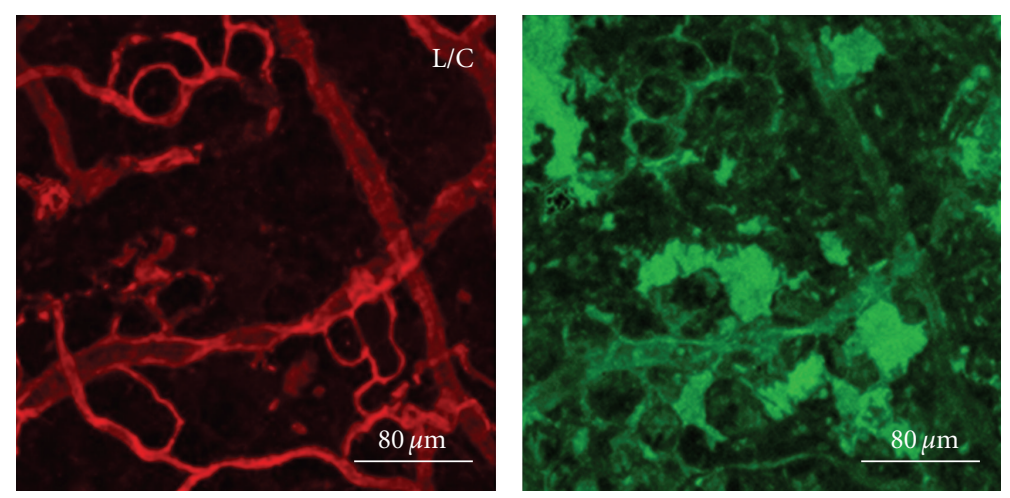

(c)
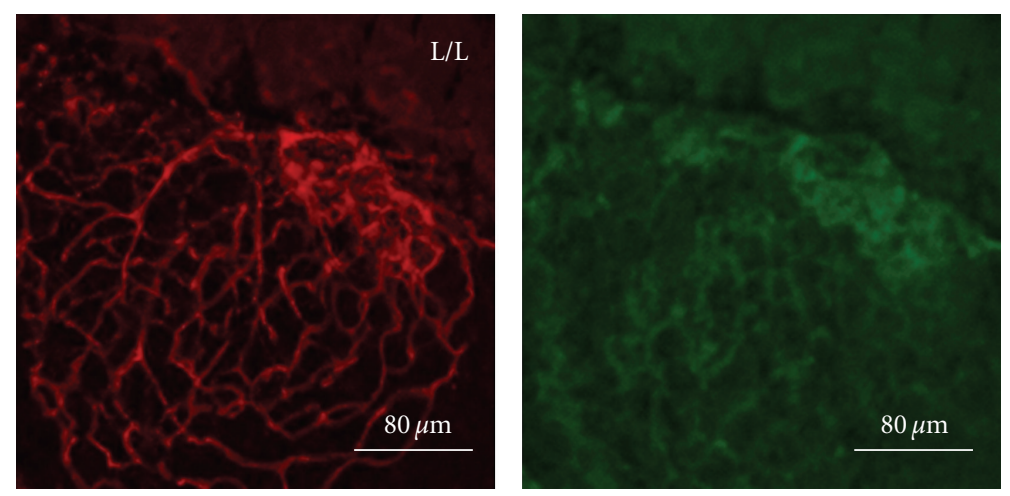

(d)

FIGURE 4: The comparison between red fluorescence images (left) excited by He-Ne laser and green fluorescence images (right) excited by Ar-Kr laser. (a) The leukocyte and the endothelial cells are both treated with buffer. (b) The leukocyte is treated with buffer and then mixed with the endothelial cells which are treated with $10 \mathrm{mg} / \mathrm{mL}$ native LDL solution. (c) The leukocyte is treated with $10 \mathrm{mg} / \mathrm{mL}$ native $\mathrm{LDL}$ solution and then mixed with the buffer-treated endothelial cells. (d) The leukocyte and the endothelial cells are both treated with $10 \mathrm{mg} / \mathrm{mL}$ native LDL solution. 
VECAD mediates the ability of leukocytes to go through the endothelial cells. Furthermore, it is considered to be associated with the dysfunction of endothelial cells and can accelerate atherosclerosis [31]. The confocal microscopic system is a useful tool for the measurements of biological properties with fluorescence detection of VECAD. It provides real-time imaging while the leukocytes are transporting through endothelial cells in the environment of tissueculturing dishes and can observe the change of VECAD concentration immediately. Although our result shows only the in vitro confocal images, it still offers a good feasibility for cardiac endoscopy with fiber optics in clinical application.

\section{Acknowledgments}

This paper was supported by the National Science Council of Taiwan under Grants NSC 99-2221-E-010-011, NSC 99-2622-E-010-001-CC3, and NSC 98-2221-E-010-004 and a grant from Ministry of Education, Aim for the Top University Plan in Yang-Ming University.

\section{References}

[1] R. H. Webb, "Confocal optical microscopy," Reports on Progress in Physics, vol. 59, no. 3, pp. 427-471, 1996.

[2] C. Smithpeter, A. Dunn, R. Drezek, T. Collier, and R. RichardsKortum, "Near real time confocal microscopy of cultured amelanotic cells: sources of signal, contrast agents and limits of contrast," Journal of Biomedical Optics, vol. 3, no. 4, pp. 429436, 1998.

[3] B. R. Masters, "Three-dimensional confocal microscopy of the human optic nerve in vivo," Optics Express, vol. 3, no. 10, pp. 356-359, 1998.

[4] A. Parrish, E. Halama, M. T. Tilli, M. Freedman, and P. A. Furth, "Reflectance confocal microscopy for characterization of mammary ductal structures and development of neoplasia in genetically engineered mouse models of breast cancer," Journal of Biomedical Optics, vol. 10, no. 5, Article ID 051602, 2005.

[5] W. F. J. Vermaas, J. A. Timlin, H. D. T. Jones et al., "In vivo hyperspectral confocal fluorescence imaging to determine pigment localization and distribution in cyanobacterial cells," Proceedings of the National Academy of Sciences of the United States of America, vol. 105, no. 10, pp. 4050-4055, 2008.

[6] P. M. Lane, S. Lam, A. McWilliams, J. C. Leriche, M. W. Anderson, and C. E. Macaulay, "Confocal fluorescence microendoscopy of bronchial epithelium," Journal of Biomedical Optics, vol. 14, no. 2, Article ID 024008, 2009.

[7] L. Thiberville, S. Moreno-Swirc, T. Vercauteren, E. Peltier, C. Cavé, and G. B. Heckly, "In vivo imaging of the bronchial wall microstructure using fibered confocal fluorescence microscopy," American Journal of Respiratory and Critical Care Medicine, vol. 175, no. 1, pp. 22-31, 2007.

[8] K. Venkatesh, M. Cohen, C. Evans et al., "Feasibility of confocal endomicroscopy in the diagnosis of pediatric gastrointestinal disorders," World Journal of Gastroenterology, vol. 15, no. 18, pp. 2214-2219, 2009.

[9] L. Thiberville, M. Saiaiin, S. Lachkar et al., "Human in vivo fluorescence microimaging of the alveolar ducts and sacs during bronchoscopy," European Respiratory Journal, vol. 33, no. 5, pp. 974-985, 2009.
[10] L. Thiberville, M. Salaün, S. Lachkar et al., "Confocal fluorescence endomicroscopy of the human airways," Proceedings of the American Thoracic Society, vol. 6, no. 5, pp. 444-449, 2009.

[11] R. Ross, "The pathogenesis of atherosclerosis: a perspective for the 1990s," Nature, vol. 362, no. 6423, pp. 801-809, 1993.

[12] F. Calara, P. Dimayuga, A. Niemann et al., "An animal model to study local oxidation of LDL and its biological effects in the arterial wall," Arteriosclerosis, Thrombosis, and Vascular Biology, vol. 18, no. 6, pp. 884-893, 1998.

[13] Á. Álvarez, M. Cerdá-Nicolás, Y. N. Abu Nabah et al., "Direct evidence of leukocyte adhesion in arterioles by angiotensin II," Blood, vol. 104, no. 2, pp. 402-408, 2004.

[14] D. S. Gareau, P. R. Bargo, W. A. Horton, and S. L. Jacques, "Confocal fluorescence spectroscopy of subcutaneous cartilage expressing green fluorescent protein versus cutaneous collagen autofluorescence," Journal of Biomedical Optics, vol. 9, no. 2, pp. 254-258, 2004.

[15] C. Saloma, C. Palmes-Saloma, and H. Kondoh, "Site-specific confocal fluorescence imaging of biological microstructures in a turbid medium," Physics in Medicine and Biology, vol. 43, no. 6, pp. 1741-1759, 1998.

[16] D. Nyqvist, M. Köhler, H. Wahlstedt, and P. O. Berggren, "Donor islet endothelial cells participate in formation of functional vessels within pancreatic islet grafts," Diabetes, vol. 54, no. 8, pp. 2287-2293, 2005.

[17] A. Eliasson Lantz, P. Jørgensen, E. Poulsen, C. Lindemann, and L. Olsson, "Determination of cell mass and polymyxin using multi-wavelength fluorescence," Journal of Biotechnology, vol. 121, no. 4, pp. 544-554, 2006.

[18] H. Swerdlow, J. Z. Zhang, D. Y. Chen et al., "Three DNA sequencing methods using capillary gel electrophoresis and laser-induced fluorescence," Analytical Chemistry, vol. 63, no. 24, pp. 2835-2841, 1991.

[19] S. Marose, C. Lindemann, and T. Scheper, "Two-dimensional fluorescence spectroscopy: a new tool for on-line bioprocess monitoring," Biotechnology Progress, vol. 14, no. 1, pp. 63-74, 1998.

[20] R. Kassies, A. Lenferink, I. Segers-Nolten, and C. Otto, "Prism-based excitation wavelength selection for multicolor fluorescence coincidence measurements," Applied Optics, vol. 44, no. 6, pp. 893-897, 2005.

[21] C. Blum, A. P. Mosk, C. Otto, W. L. Vos, and V. Subramaniam, "Spectral emission imaging to map photonic properties below the crystal surface of 3D photonic crystalsll," Journal of the Optical Society of America B, vol. 26, no. 11, pp. 2101-2108, 2009.

[22] Y. Shao, J. Qu, H. Li et al., "High-speed spectrally resolved multifocal multiphoton microscopy," Applied Physics B, vol. 99, pp. 633-637, 2010.

[23] V. Seyfried, H. Birk, R. Storz, and H. Ulrich, "Advances in multispectral confocal imaging," in Confocal, Multiphoton, and Nonlinear Microscopic Imaging, Proceedings of SPIE, pp. 147-157, June 2003.

[24] M. M. Mahfouz and F. A. Kummerow, "Oxidized low density lipoprotein inhibits prostacyclin generation by rat aorta in vitro: a key role of lysolecithin," Prostaglandins and Other Lipid Mediators, vol. 66, no. 4, pp. 283-304, 2001.

[25] I. S. Young and J. McEneny, "Lipoprotein oxidation and atherosclerosis," Biochemical Society Transactions, vol. 29, no. 2, pp. 358-362, 2001.

[26] M. Yanagisawa, A. Inoue, T. Ishikawa et al., "Primary structure, synthesis, and biological activity of rat endothelin, an endothelium-derived vasoconstrictor peptide," Proceedings of the National Academy of Sciences of the United States of America, vol. 85, no. 18, pp. 6964-6967, 1988. 
[27] L. Gao, K. Chalupsky, E. Stefani, and H. Cai, "Mechanistic insights into folic acid-dependent vascular protection: dihydrofolate reductase (DHFR)-mediated reduction in oxidant stress in endothelial cells and angiotensin II-infused mice: a novel HPLC-based fluorescent assay for DHFR activity," Journal of Molecular and Cellular Cardiology, vol. 47, no. 6, pp. 752-760, 2009.

[28] P. L. Hordijk, E. Anthony, F. P. J. Mul, R. Rientsma, L. C. J. M. Oomen, and D. Roos, "Vascular-endothelial-cadherin modulates endothelial monolayer permeability," Journal of Cell Science, vol. 112, no. 12, pp. 1915-1923, 1999.

[29] H. Koga, S. Sugiyama, K. Kugiyama et al., "Elevated levels of VE-cadherin-positive endothelial microparticles in patients with type 2 diabetes mellitus and coronary artery disease," Journal of the American College of Cardiology, vol. 45, no. 10, pp. 1622-1630, 2005.

[30] F. Breviario, L. Caveda, M. Corada et al., "Functional properties of human vascular endothelial cadherin (7B4/cadherin-5), an endothelium-specific cadherin," Arteriosclerosis, Thrombosis, and Vascular Biology, vol. 15, no. 8, pp. 1229-1239, 1995.

[31] E. Dejana, "Endothelial adherens junctions. Implications in the control of vascular permeability and angiogenesis," Journal of Clinical Investigation, vol. 98, no. 9, pp. 1949-1953, 1996. 


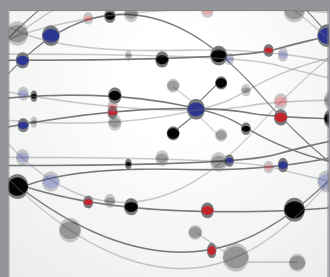

The Scientific World Journal
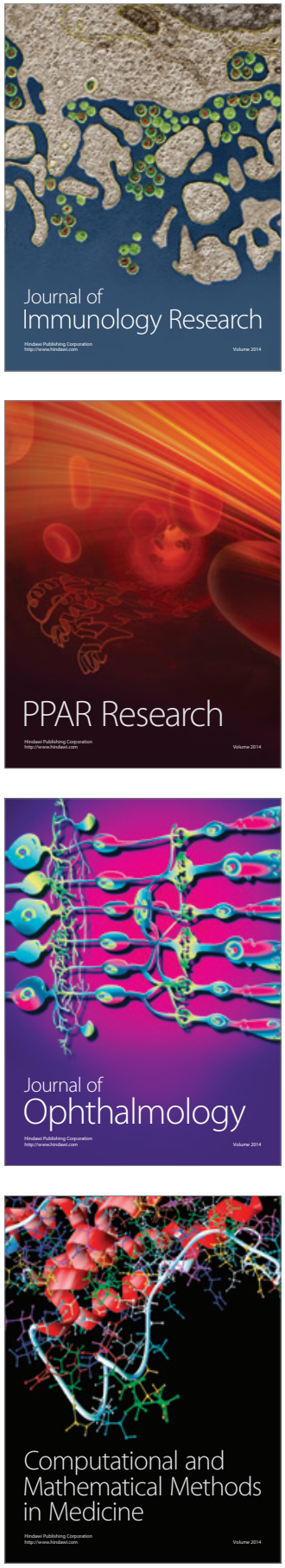

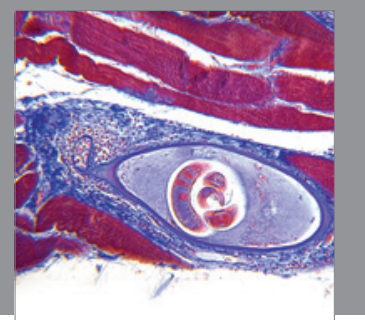

Gastroenterology

Research and Practice
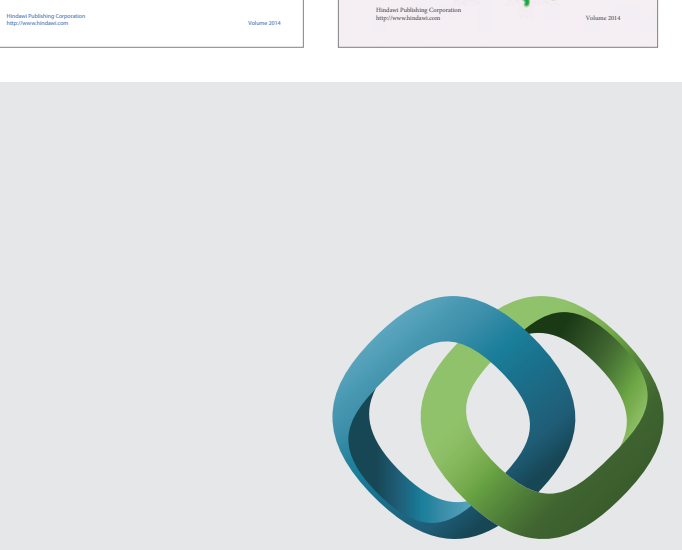

\section{Hindawi}

Submit your manuscripts at

http://www.hindawi.com
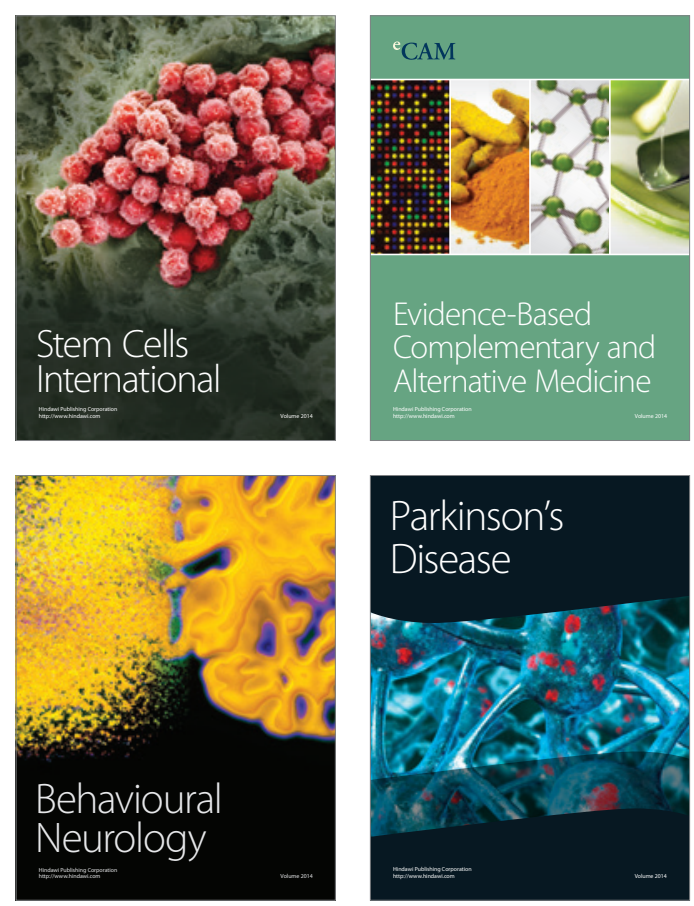

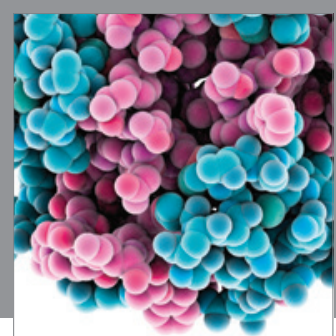

Journal of
Diabetes Research

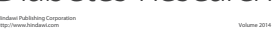

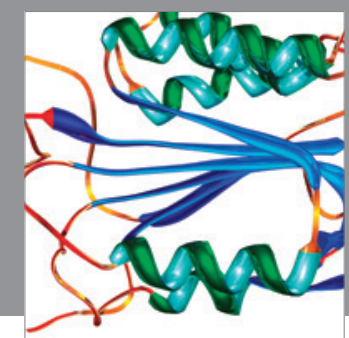

Disease Markers
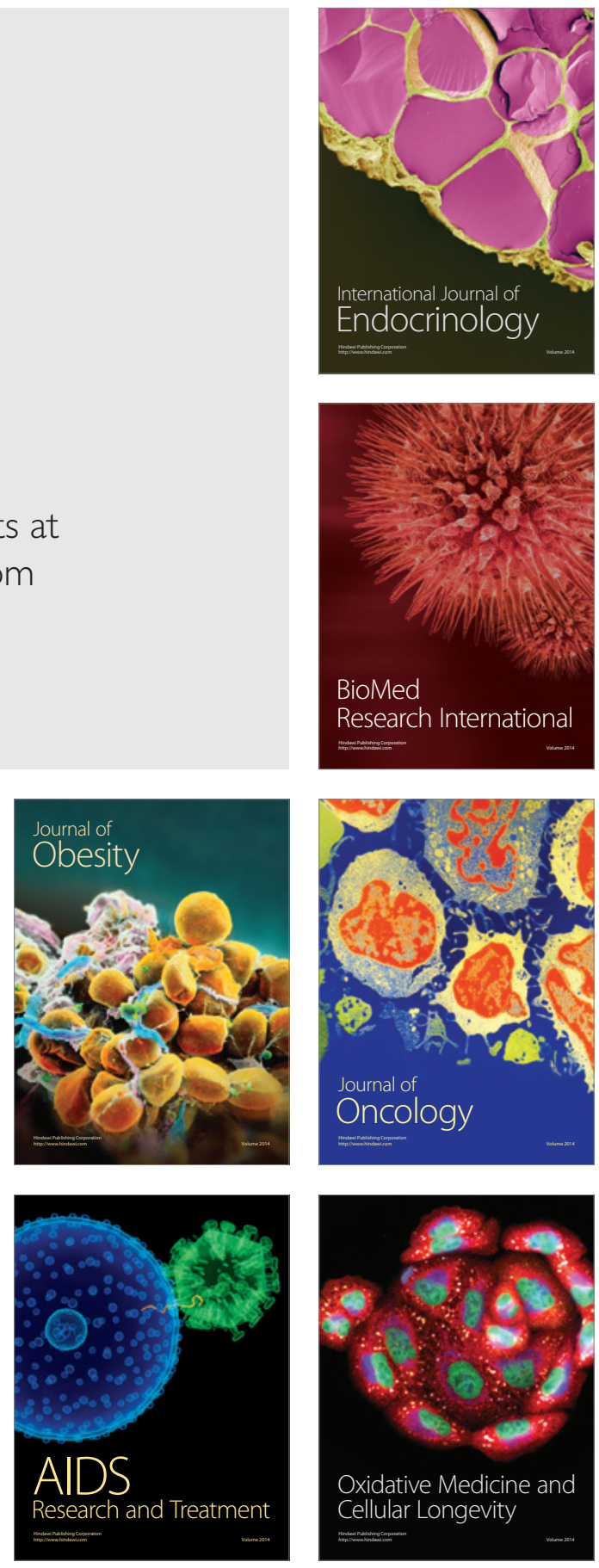